\title{
Living-related limbal allograft transplantation for bilateral stem cell deficiency in a patient with bilateral chemical burn
}

\author{
Anastasia Tasiopoulou, Ioannis A. Mallias \\ Mediterraneo Hospital, Glyfada, Athens, Greece
}

\begin{abstract}
INTRODUCTION. The purpose of this paper was to report the long-term outcome of a living-related limbal allograft transplantation followed by penetrating keratoplasty in a patient with severe bilateral stem cell deficiency.

MATERIALS AND METHODS. A 50-year-old woman presented with extensive bilateral stem cell deficiency secondary to ocular acid burn on both eyes. Visual acuity was counting fingers at $30 \mathrm{~cm}$ on the right eye and counting fingers at $1 \mathrm{~m}$ Snellen line on the left. Limbal allograft transplantation on the right eye from a first-degree living-related relative was performed. The recipient was prepared by removing the abnormal corneal epithelium and vascularised pannus of the right eye under general anaesthesia. Two limbal allografts (mean length of 2-3 corresponding anatomic positions on the recipient). Postoperatively, the host received systemic immunosuppression (steroids and cyclosporine). RESULTS. The cornea of the right eye achieved reepithelialisation within two weeks after surgery, followed by reduction in vascularity in eight weeks time. After one year of regular follow up, the corneal surface remained stable whereas the stroma was opaque and the vision was low. Therefore, the patient underwent penetrating keratoplasty on the same eye. After the surgery the corneal graft was clear and the visual acuity on the right eye improved to 20/50 Snellen line. No graft rejection occurred after a five-year follow-up.

CONCLUSION. Living-related limbal allograft transplantation is a good alternative option in cases of total bilateral stem cell deficiency whenever a conjunctival limbal autograft transplantation is not possible.
\end{abstract}

KEY WORDS: living-related limbal allograft transplantation, stem cell deficiency, bilateral chemical burn

Ophthalmol J 2016; Vol. 1, No. 2, 47-51

\section{INTRODUCTION}

Limbal stem cells are responsible for the homeostasis of the corneal epithelium and probably reside in the basal layer of the palisades of Vogt [1]. When there is need for tissue regeneration, the stem cells start to divide $[2,3]$. The basal limbal epithelium contains the unique and least differentiated cells of corneal epithelium [4]. In the case of limbal stem cell dysfunction or deficiency due to injury, the conjunctiva must reepithelialise the corneal surface. This process is prolonged and does not allow the production of a normal phenotypic corneal epithelium [5]. Limbal stem cell deficiency can be defined as primary and secondary. Primary deficient states are caused by an insufficient stromal microenvironment to keratitis associated with multiple endocrine deficiencies and neurotrophic keratopathy. Secondary states are more common than primary ones and include external factors destroying limbal stem cells, such as chemical or thermal injuries, Stevens-Johnson syndrome, ocular cicatricial pemphigoid, multiple surgeries or cryotherapies, contact lens overuse, or extensive microbial infection $[2,3,6,7]$. The most common among the acquired states are chemical or thermal injuries due to alkali or acid compounds [8]. As proposed by Kenyon and Tseng, in patients with total 
limbal stem cell deficiency, limbal autograft or allograft transplantation are indicated for corneal surface reconstruction. This may be combined with or followed by keratoplasty and/or amniotic membrane transplantation [9]. Conjunctival autograft (CAU) or conjunctival limbal autograft (CLAU) could be considered [8]. In cases of bilateral disease where limbal stem cell autografting is precluded, the only option for stem cell restoration is the use of allogeneic sources $[10,11]$. These sources include cadaveric keratolimbal allograft (KLAL), living-related conjunctival limbal allograft (lr CLAL), and ex vivo stem cell allograft expansion [12].

\section{MATERIALS AND METHODS}

A 50-year-old woman presented with ocular acid burn on both eyes in September 2008. The average time between first presentation and the initial burn was almost six months. She had a medical history of diabetes mellitus type II and was under insulin treatment. Visual acuity was counting fingers at $30 \mathrm{~cm}$ on the right eye and counting fingers at $1 \mathrm{~m}$ Snellen line on the left. The intraocular pressure was $11 \mathrm{~mm} \mathrm{Hg}$ on the right eye and $14 \mathrm{~mm} \mathrm{Hg}$ on the left with Goldmann applanation tonometry. Slit lamp examination revealed no lid abnormalities or formation of symblepharon between surfaces of the palpebral and bulbar conjunctiva. The cornea was opacified and the conjunctival epithelium covered the anterior surface of the cornea due to extensive stem cell deficiency. Also there was superficial and deep corneal vascularisation $\left(270^{\circ}\right.$ on the right and one $180^{\circ}$ on the left) with vessels extending almost $5 \mathrm{~mm}$ from the limbus into the centre of the cornea (Fig. 1). Papillary reflexes were normal. Fundus was normal on both eyes, although fundoscopy was difficult due to corneal opacification; therefore, the use of limbal stem cell autografts was not possible, and lr CLAL on the right eye was performed.

HLA typing was used in order to choose the ideal living-related donor to prevent stem cell rejection. The sister of the patient was the best living HLA- matched donor. Both of her eyes were evaluated to ensure there was no underlying ocular history and the non-dominant eye (the left one) was chosen for the donation. In order to exclude the presence of glaucoma, the donor underwent evaluation of the optic nerves with OCT and tonometry, which were normal. Also she was screened for bloodborne diseases (hepatitis B and C) and immunodeficiency virus (HIV).

Prior to the operation, the nature of the surgical procedure and all surgical risks were explained to the patient and the donor and informed consents were signed by both of them. The donor was operated using retrobulbar

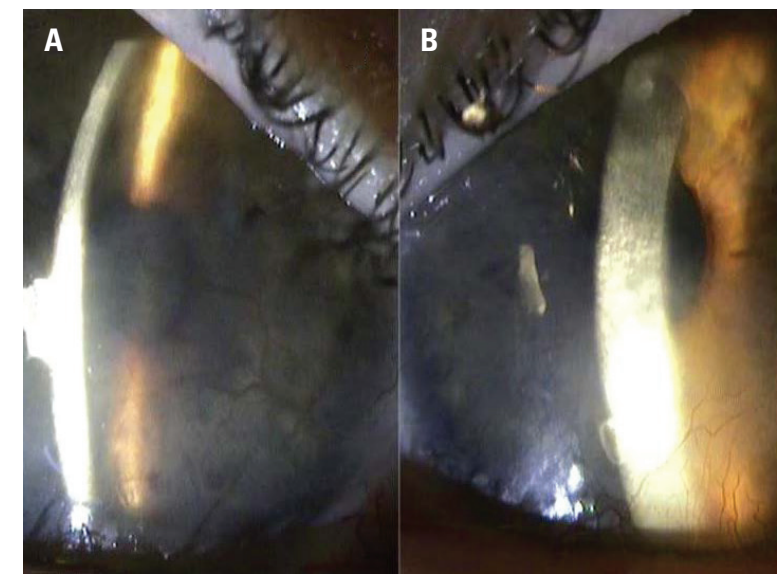

FIGURE 1. View of the anterior segment of the patient on initial examination: A. Right eye: conjuctivalisation of the cornea with $270^{\circ}$ superficial and deep corneal vascularisation, B. Left eye: conjunctival epithelium covering the cornea with $180^{\circ}$ vascularisation

anesthesia and sedation whereas the patient was operated under general anaesthesia. Surgery on the recipient was performed following surgery on the donor.

\section{SURGICAL TECHNIQUE}

Surgery was performed by the second author Dr. Ioannis A. Mallias. On the donor's left eye the sites for donation were marked with a gentian violet pen and incorporated within the donor tissue. Two separate limbal allografts (mean length of 2-3 clock hours) were resected from the 12 o'clock and 6 o'clock areas. The posterior edge was cut $5 \mathrm{~mm}$ posterior to the limbus and the tissue was reflected on to the corneal surface. The remaining conjunctiva of the donor was anchored to the sclera with two 8-0 Vicryl sutures with instillation of a steroid antibiotic ointment, and the eye was patched until the following day. 1:10,000 topical epinephrine was used for haemostasis. A $360^{\circ}$ limbal conjunctival peritomy was performed. Recipient beds of three clock hours at the 12 and 6 o'clock meridian and to $5 \mathrm{~mm}$ behind the limbus were created, and the abnormal corneal epithelium and vascularised pannus were removed.

The harvested allografts were then transferred to corresponding anatomic positions on the recipients with the limbal edge at the recipient limbus and secured with 10-0 nylon sutures. Extensive care was taken to suture the grafts properly (not upside down) and to make sure that the limbal edge of the graft was sutured on the limbal site of the recipient. At the end of the procedure, a steroid antibiotic ointment was instilled and the eye was patched until the following day [12]. Postoperatively, the donor received antibiotic and steroid drops four times daily. The 
former were used for five days and the latter were gradually tapered over a period of three weeks. The recipient was managed postoperatively with topical and systemic treatment. Topical treatment included dexamethasone sodium phosphate $(0.1 \%)$, ofloxacin $(0.5 \%)$, and preservative-free tear supplements, each four times daily. Topical corticosteroids were gradually tapered over a period of four months, and topical antibiotics were discontinued two weeks after surgery. However, tear supplements were maintained and the eye was patched all the time for twenty days, and it was only opened in order to instil the drops. Systemic treatment included immunosuppression with steroids and cyclosporine A. Intravenous methyl prednisolone $2 \mathrm{mg} / \mathrm{kg} /$ day was commenced on the day of surgery and maintained for three additional days. Oral prednisolone $1 \mathrm{mg} / \mathrm{kg} /$ day was then started for one week and tapered over two months and systemic cyclosporin A $4 \mathrm{mg} / \mathrm{kg} /$ day separated in two doses per day was administered for one year. While on treatment, the patient was followed by her rheumatologist for possible side effects of the systemic immunosuppression, and she underwent periodic evaluation of creatinine and blood pressure.

\section{RESULTS}

The keratolimbal allografts were vascularised normally during the first postoperative days without signs of tissue necrosis or rejection. The cornea of the right eye achieved reepithelialisation within two weeks after surgery. The epithelial healing was complete with normal corneal epithelium and no fluorescent staining was observed. In eight weeks' time, there was regression of peripheral neovascularisation on the right eye (Fig. 2). The cornea was covered with normal corneal epithelium, but there was significant haziness of the corneal stroma which limited the best corrected visual acuity (BCVA) to 20/200 Snellen line. After one year of regular follow up, the corneal surface remained stable and no vascularisation of the peripheral cornea was observed. However, the stroma was opaque and the visual acuity had no further improvement. In order to improve the vision, the patient underwent penetrating keratoplasty on the same eye in October 2009. The surgery was performed under general anaesthesia and $8-\mathrm{mm}$ trephination of the host cornea was done during the operation. Then $8.25 \mathrm{~mm}$ trephination of the donor cornea was performed and the donor button was secured in the host bed with sixteen interrupted 10-0 nylon sutures (Fig. 3).

Postoperatively, the patient received ofloxacin drops $(0.5 \%)$ four times a day for two weeks and topical corticosteroids (dexamethasone sodium phosphate month). Oral cyclosporine A ( $4 \mathrm{mg} / \mathrm{kg} /$ day) was also administered
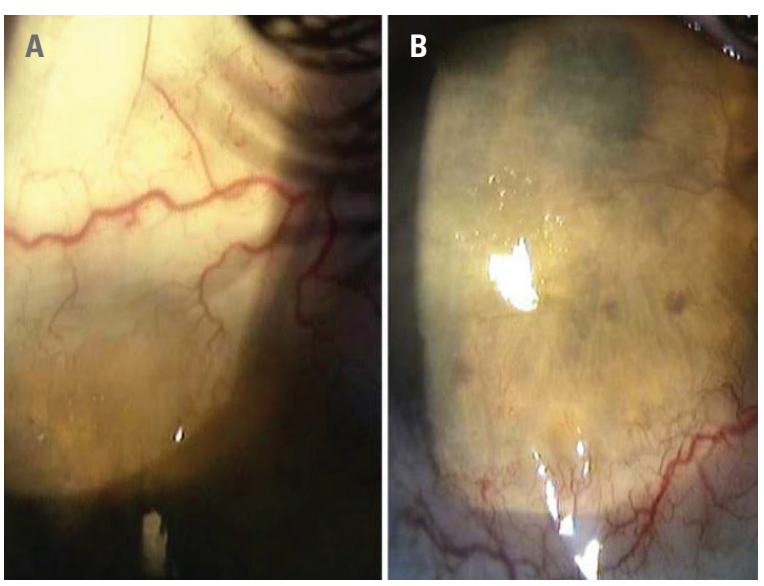

FIGURE 2. View of the right eye of the patient eight weeks after living-related limbal allograft transplantation. Regression of peripheral neovascularisation: A. Superior keratolimbal allograft, B. Inferior keratolimbal allograft

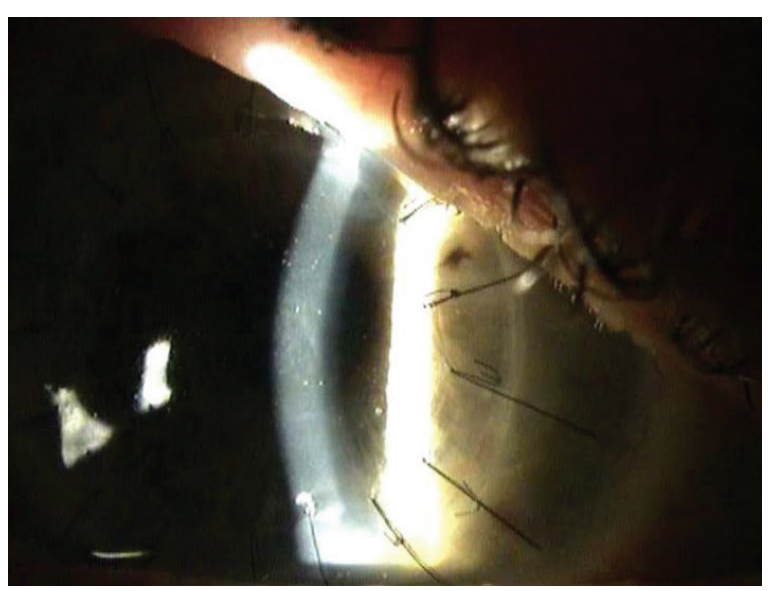

FIGURE 3. View of the right eye one week after penetrating keratoplasty. The cornea is clear, covered with normal corneal epithelium with no signs of allograft rejection

for six months. Additionally, regular corneal topographies were performed in order to assess the postoperative astigmatism. Selective suture removal started after the completion of the third postoperative month. The graft remained clear with normal corneal epithelium during the follow-up period, which was four years (Fig. 4). The Snellen visual acuity was 20/50 with spectacles and 20/30 with gas permeable contact lenses.

\section{DISCUSSION}

The appropriate management of a patient with severe stem cell deficiency due to chemical burn requires careful assessment of factors such as laterality of disease, severity of the injury, and presence of conjunctival inflammation.

When the ocular surface injury is unilateral and there is total stem cell deficiency, then a keratolimbal autograft 


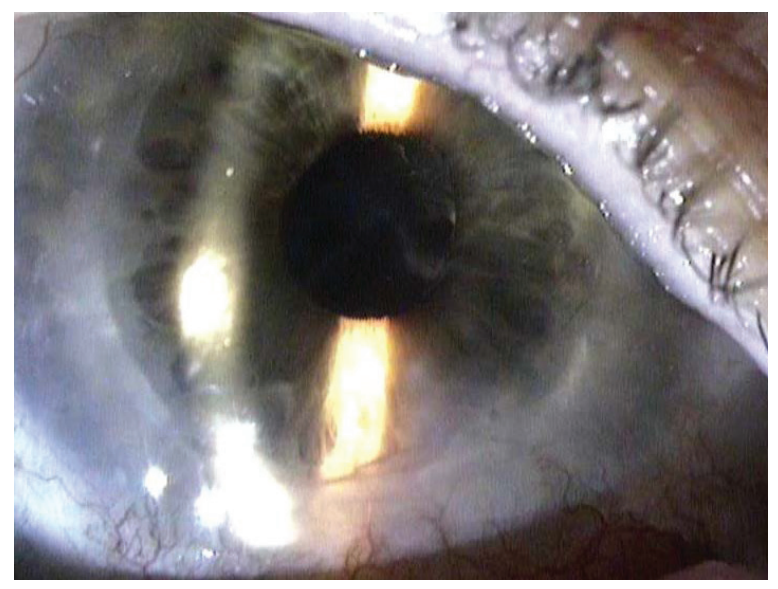

FIGURE 4. Four years after penetrating keratoplasty of the right eye, the graft has remained clear and the visual acuity has significantly improved

from the healthy fellow eye is the most suitable treatment option. This treatment option can be combined with the use of an amniotic membrane. However, the worst prognostic category is bilateral limbal stem cell deficiency accompanied by conjunctival inflammation, such as in our case. The first choice of treatment in these patients is lr CLAL. If a living relative is not available, then KLAL is the next option. Ex vivo stem cell expansion may also be employed in such cases [13]. Evaluating the technique of lr CLAL, there is the advantage of providing some degree of immune histocompatibility [12, 14, 15]. Therefore, the possibility of graft rejection is much lower as well as the need for systemic immunosuppression. Furthermore, there is a reduction of the cell death rate due to obvious disadvantages in using living related limbal allografts because limbal allografting is limited by the amount of tissue that can be transplanted. In particular, a superior limbal graft length of five or six clock hours is a limitation in order to protect the donor's eye $[12,16]$. Whereas, in KLAL a complete limbus for transplantation and thus a larger load of stem cells with restoration of a barrier to the whole limbus can be obtained from a cadaveric donor [13].

The chemical burn of our patient was bilateral and the consequent stem cell deficiency was total. She underwent an Ir CLAL on her right eye with two limbal grafts from her sister's eye, who was the best living HLA-matched donor. The grafts were protected by systemic immunosuppression with steroids and cyclosporine A. With this procedure the corneal epithelium was reconstructed and the visual acuity was improved. After a five-year follow-up, no graft rejection occurred, although the patient was under systemic immunosuppression only for the first year. However, Huang et al. [17] reported that partial limbal stem cell deficiency may be the best indication for lr CLAL, because even if graft rejection were to occur, the amount of residual stem cells would be able to sustain sufficient epithelial cell production. They performed this procedure in eyes with partial limbal deficiency $(<50 \%)$ secondary to ocular chemical burns. However, they did not administer systemic immunosuppression with cyclosporine A, but they accomplished a lower rejection rate compared with rejection rates of other studies. Conversely, Rao et al. [16] and Daya et al. [15] emphasised the importance of immunosuppression, stating that systemic immunosuppressants are necessary even if the donors are compatible to recipients.

As far as HLA compatibility is concerned, Kwitko et al. [14] reported that HLA matched limbal allograft transplantation provides long-term graft survival and minimises the rate of graft rejection. The importance of a good HLA matching is also highlighted by Daya et al. [15] and other studies [11]. Whereas, the Collaborative Corneal Transplantation Studies Research Group demonstrated that HLA antigen matching does not ensure the survival of corneal graft in high-risk corneal transplantation [18].

An alternative option to limbal allograft transplantation is the Cincinnati procedure performed by Biber et al. [19]. In this procedure, patients with severe ocular surface disease and conjunctival deficiency underwent combined 1r CLAL and KLAL and achieved significant improvement in visual acuity.

A study conducted by Sonmez et al. [20] is also worth mentioning. In this study, they performed either KLAL combined with lr CLAL or KLAL alone, using only fibrin sealant in order to secure the allografts to the recipient's ocular surface without sutures. They reported that the use of fibrin tissue glue decreased the operation time and suture-related complications such as postoperative inflammation and graft rejection. In our case, although we used sutures to secure the allografts to the recipient's eye, we did not observe excessive inflammation or graft rejection. Moreover, we achieved a five-year survival of the limbal allografts by using good HLA matching and systemic immunosuppression. Hence, we would like to emphasise the importance of these two factors in the long-term survival of limbal allografts, especially in cases of total limbal stem cell deficiency.

\section{CONFLICT OF INTEREST}

None declared.

\section{REFERENCES}

1. Davanger $M$, Evensen $A$. Role of the pericorneal papillary structure in renewal of renewal of corneal epithelium. Nature 1971; 229: 560-61.

2. Tseng SCG. Concept and application of limbal stem cells. Eye 1989; 57: 201-209. 
3. Kruse FE. Stem cells and corneal epithelial regeneration. Eye 1994; 8: 170-183

4. Kinoshit S, Friend J, Kiorpes TC et al. Keratin like proteins in corneal and conjunctival epithelium are different. Invest Ophthalmol Vis Sci 1983; 24: 577-581.

5. Shapiro M, Friend J, Thoft R. Corneal re-epithelialization from the conjunctiva. Invest Ophthalmol Vis Sci 1991; 32: 109-113.

6. Pfister RR. Corneal stem cell disease: Concepts, categorization, and treatment by auto and homotransplantation of limbal stem cells. CLAO J 1994; 20: 64-72.

7. Dua HS. Stem cells of the ocular surface: Scientific principles and clinical applications. Br J Ophthalmol 1995; 79: 968-969.

8. Kim T, Khosla-Gupta BA. Chemical and thermal injuries to the ocular surface. In: Holland EJ, Mannis MJ (eds.). Ocular surface disease. Medical and Surgical Management. Springer, New York 2002: 100, 108, 109.

9. Kenyon KR, Tseng SCG. Limbal autograft transplantation for ocular surface disorders. Ophthalmology 1989; 96: 709-723.

10. Tseng SC. Classification of conjunctival surgeries for corneal diseases based on stem cell concept. Ophthalmol Clin North Am 1990; 3: 595-610.

11. Holland EJ, Schwartz GS. The evolution of epithelial transplantation for severe ocular surface disease and a proposed classification system. Cornea 1996; 15: 549-556.

12. Daya SM, Holland EJ, Mannis MJ. Living-related conjunctival limbal allograft. In: Holland EJ, Mannis MJ (eds.). Ocular surface disease. Medical and Surgical Management. Springer, New York 2002: 201.
13. Holland EJ, Mannis MJ, Schwartz GS. Developing a logical paradigm for the clinical management of severe ocular surface disease. In: Holland EJ, Mannis MJ (eds.). Ocular surface disease. Medical and Surgical Management. Springer, New York 2002: 269-271.

14. Kwitko $S$, Marinho D, Barcaro $S$, et al. Allograft conjunctival transplantation for bilateral ocular surface disorders. Ophthalmology 1995; 102: 1020-1025.

15. Daya SM, llari L. Living related conjunctival limbal allograft for the treatment of stem cell deficiency. Ophthalmology 2001; 108: 126-133.

16. Rao SK, Rajagopal R, Sitalakshmi G et al. Limbal allografting from related live donors for corneal surface reconstruction. Ophthalmology 1999; 106: 822-828.

17. Huang $T$, Wang $Y$, Zhang $H$ et al. Limbal allografting from living-related donors to treat partial limbal deficiency secondary to ocular chemical burns. Arch Ophthalmol 2011; 129: 1267-1273.

18. The collaborative corneal transplantation studies (CCTS). Effectiveness of histocompatibility matching in high-risk corneal Research Group. Arch Ophthalmol 1992; 110: 1392-1403.

19. Biber JM, Skeens HM, Neff KD et al. The cincinnati procedure: technique and outcomes of combined living-related conjunctival limbal allografts and keratolimbal allografts in severe ocular surface failure. Cornea 2011; 30: 765-771.

20. Sonmez B, Beden U. Fibrin glue-assisted sutureless limbal stem cell transplantation surgery for the treatment of severe ocular chemical injury. Cornea 2011; 30: 296-300. 
